\title{
Field emission characteristics of a graphite nanoneedle cathode and its application to scanning electron microscopy
}

\author{
Yoichiro Neo and Hidenori Mimura \\ The Graduate School of Electronic Science and Technology, Shizuoka University, 3-5-1 Johoku, Hamamatsu \\ 432-8011, Japan \\ Takahiro Matsumoto ${ }^{\text {a) }}$ \\ Research and Development Center, Stanley Electric Corporation, 5-9-5 Tokodai, Tsukuba 300-2635, Japan \\ and Research Institute of Electronics, Shizuoka University, 3-5-1 Johoku, Hamamatsu 432-8011, \\ Japan
}

(Received 6 October 2005; accepted 6 January 2006; published online 16 February 2006)

\begin{abstract}
A high-brightness electron beam of more than $10^{11} \mathrm{~A} \mathrm{sr}^{-1} \mathrm{~m}^{-2}$ was achieved from a graphite nanoneedle cathode, which was fabricated by simple hydrogen plasma etching of a graphite rod. A field emission was obtained at a high residual pressure of $10^{-6}$ Torr. The performance of this cold cathode was demonstrated by the fabrication of a scanning electron microscope, which was operated at a high residual pressure of $10^{-5}-10^{-6}$ Torr. The brightness of this cathode offers a convenient field electron emission source that does not require a massive ultrahigh vacuum system. (C) 2006 American Institute of Physics. [DOI: 10.1063/1.2173712]
\end{abstract}

Carbon cathodes, such as carbon nanotubes (CNTs), have attracted special interest as high-brightness point electron sources because of their superior emission characteristics. $^{1,2}$ The best brightness of $10^{9} \mathrm{~A} \mathrm{sr}^{-1} \mathrm{~m}^{-2} \mathrm{~V}^{-1}$ was reported for a multiwalled $\mathrm{CNT}^{3}$ This characteristic suggests that carbon cathodes are promising for the fabrication of devices with point electron sources, such as scanning electron microscopes (SEMs) and transmission electron microscopes (TEMs).

However, CNT emitters still have problems related to adherence between the nanotubes and the supporting wires or substrates. The adherence problems lead to a drop in potential at the interface, which in turn causes saturation of electron emission and deterioration of the cold cathode at high voltage operation. ${ }^{4}$ Furthermore, a sophisticated adhesion technique is generally required to attach CNTs on metal wires or substrates. However, a solid contact for practical use has not been obtained as yet.

In this study, we develop a nanometer-sized tailored cathode that can be made by simply etching graphite nanoneedles onto a graphite rod. We found that a plurality of nanoneedles with a high aspect ratio, of the order of 100 , can be fabricated on the spearhead region of the graphite rod. The diameter of each nanoneedle is less than $100 \mathrm{~nm}$. This graphite nanoneedle cathode shows superior field emission characteristics compared to the widely researched CNTs, and does not have the adherence problem commonly seen in CNTs, thus making it promising for applications in point electron source devices. We show that high brightness, on the order of $10^{11} \mathrm{~A} \mathrm{sr}^{-1} \mathrm{~m}^{-2}$, was obtained from a graphite nanoneedle at a high residual pressure of about $10^{-6}$ Torr. The graphite nanoneedle offers a convenient point emission source that can be used to realize a high-resolution electron microscope without the need for a massive ultrahigh vacuum system. The performance of the graphite nanoneedle cathode was also demonstrated by the fabrication of a field emission SEM (FE-SEM) system.

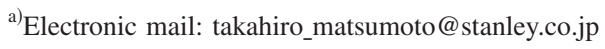

A graphite rod was mechanically sharpened at one end to less than a $10 \mu \mathrm{m} \phi$ diameter, and then graphite nanoneedles were fabricated on the tip by hydrogen plasma etching. The hydrogen plasma etching conditions were a radio frequency power of $800 \mathrm{~W}$, a gas pressure of 10 Torr, a $\mathrm{H}_{2}$ gas flow rate of $80 \mathrm{sccm}$, a substrate temperature of $600{ }^{\circ} \mathrm{C}$, a substrate bias of $-200 \mathrm{~V}$, and an etching duration of about $30 \mathrm{~min}$

Figure 1 shows a SEM image of the graphite rod surface after hydrogen plasma etching (a), a transmission electron microscope image of single graphite nanoneedle (b), and its electron diffraction pattern (c). Before the plasma etching, the mechanically sharpened graphite rod had a smooth surface. After the etching, the surface of graphite rod had a plurality of nanoneedle structures with a high aspect ratio, of the order of 100, as shown in Fig. 1(a). The plurality of graphite nanoneedles is suitable for a field emission cathode operating at high residual pressure. This is because multiple nanometer-sized emission sites ensure longevity of the cathode compared to that of a single emission site, and because the cathode with a single emission site is easily damaged by the ion bombardment inevitably occurring at high residual pressure. Magnification of the spearhead region shown in

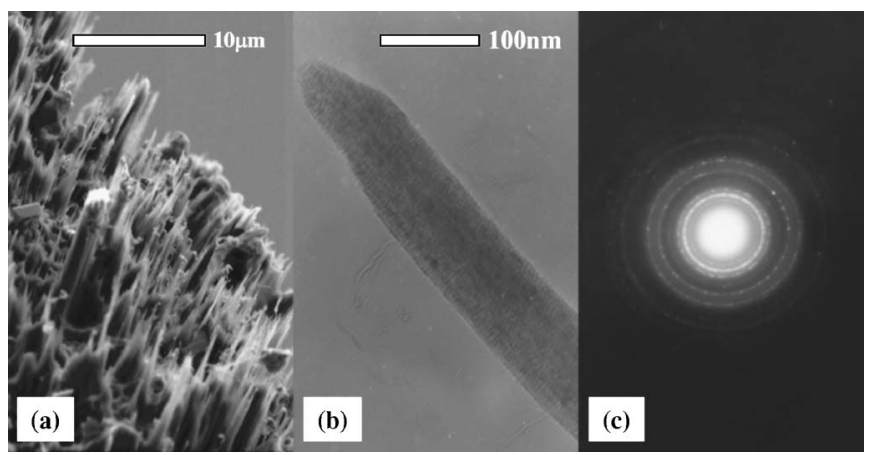

FIG. 1. (a) SEM image of a graphite rod surface after hydrogen plasma etching, (b) TEM image of a single graphite nanoneedle cathode, and (c) electron diffraction pattern of a single graphite needle. 


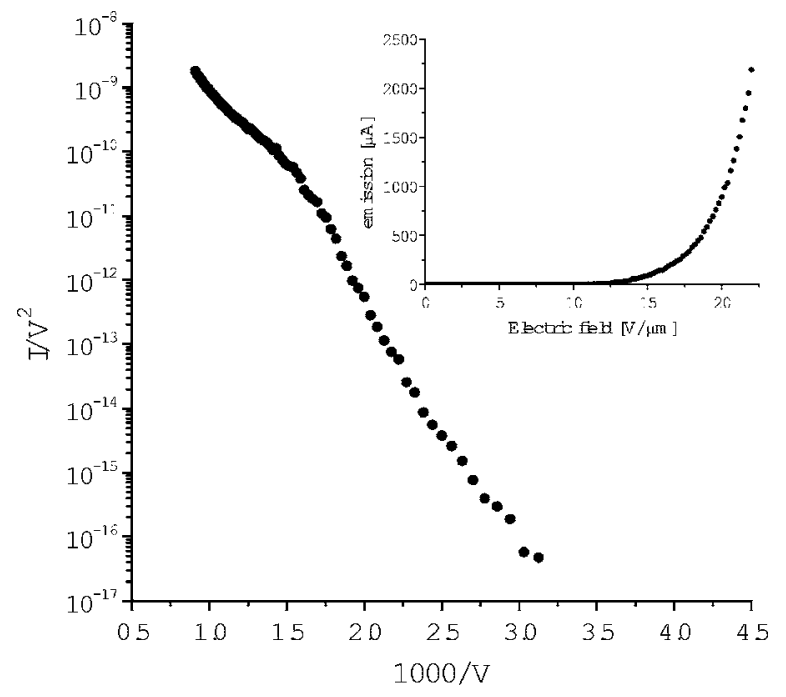

FIG. 2. Fowler-Nordheim plot of total emission characteristics from a graphite rod after etching. Inset shows typical current-voltage characteristics.

Fig. 1(b) reveals a graphite nanoneedle structure, the diameter of which was of the order of $100 \mathrm{~nm}$. The nanometersized diameter, as well as the high aspect ratio, are suitable for a point electron source such as an electron microscope. Figure 1(c) shows that the diffraction pattern is halo and ring. This pattern implies that the structure of this cathode is amorphous and/or polycrystalline. Compared to CNT, the graphite nanoneedles were obviously less ordered, and each needle has no crystalline structure.

The total emission characteristics of multi-graphite nanoneedles were determined by measuring the current collected on a $3.0 \mathrm{~mm}$ diameter aluminum anode, which was located at $50 \mu \mathrm{m}$ in front of the cathode. All the measurements were carried out in a vacuum chamber with a residual pressure of about $5 \times 10^{-7}$ Torr.

Figure 2 shows a typical Fowler-Nordheim plot of the emission current from an etched graphite rod. This plot suggests that the electron emission was dominated by the Fowler-Nordheim tunneling process. ${ }^{5}$ Based on this characteristic, the emission was caused through field emission. The inset of Fig. 2 shows typical current-voltage characteristics. The threshold of the applied electric field for the emission was about $3-8 \mathrm{~V} / \mu \mathrm{m}$ and the magnitude of the emission current exceeded $2 \mathrm{~mA}$, which is about $10^{8}$ times larger than that of the only mechanically sharpened graphite rod at $22.0 \mathrm{~V} / \mu \mathrm{m}$.

To evaluate the brightness of the single graphite nanoneedle, an FE-SEM system was fabricated. A schematic of a SEM optical system equipped with an etched graphite rod is shown in Fig. 3. The SEM experiments were performed under a residual pressure of about $2.0 \times 10^{-6}$ Torr. This pressure was much higher than that of typical FE-SEM with a tungsten tip, which generally requires a low residual pressure, below $10^{-9}$ Torr. Either an etched graphite rod or a tungsten filament thermal emission (TE) cathode was mounted on the SEM system. Electrode 1, shown in Fig. 3, was used as a wehnelt for a TE cathode and as an extracting gate electrodem $(0.1-1 \mathrm{kV})$ for a FE cathode. Other electrodes, such as a suppressor to focus the electron beam, were not included in this SEM system. A single final objective lens was used to focus the crossover on the target (sample

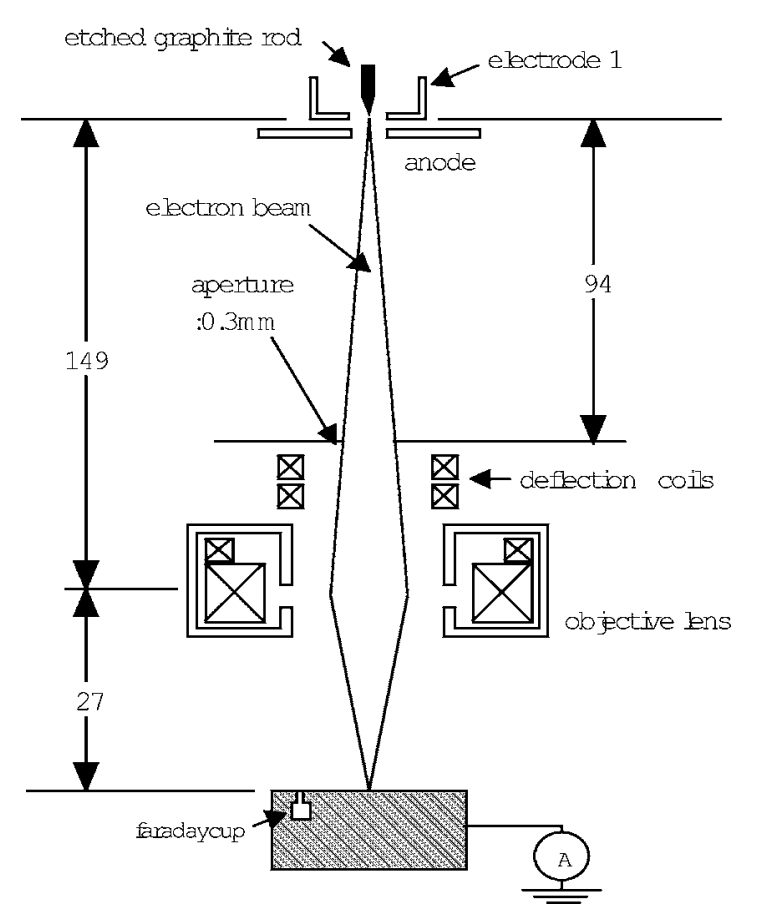

FIG. 3. Schematic of SEM system. Electrode 1 was used as a wehnelt for a $\mathrm{TE}$ cathode and as an extracting gate electrode for a FE cathode.

holder), where the electron beam diameter and convergence angle were measured. The objective lens was composed of a permanent magnet and assistant coil and was designed to focus at $3.0 \mathrm{kV}$ acceleration voltage. The aperture diameter was $0.3 \mathrm{~mm} \phi$. The spatial resolution was evaluated by obtaining the images of a $4 \mu \mathrm{m}$ wide copper mesh located on the sample holder. In this lens configuration, the source size was reduced to 0.182 times at the sample target. In addition, a Faraday cup on the sample holder was used to collect the probe current.

Figure 4(a) shows the SEM image of the copper mesh obtained using the TE cathode to compare both the resolution and brightness with those of the graphite nanoneedle. The resolution of the image obtained using the TE cathode

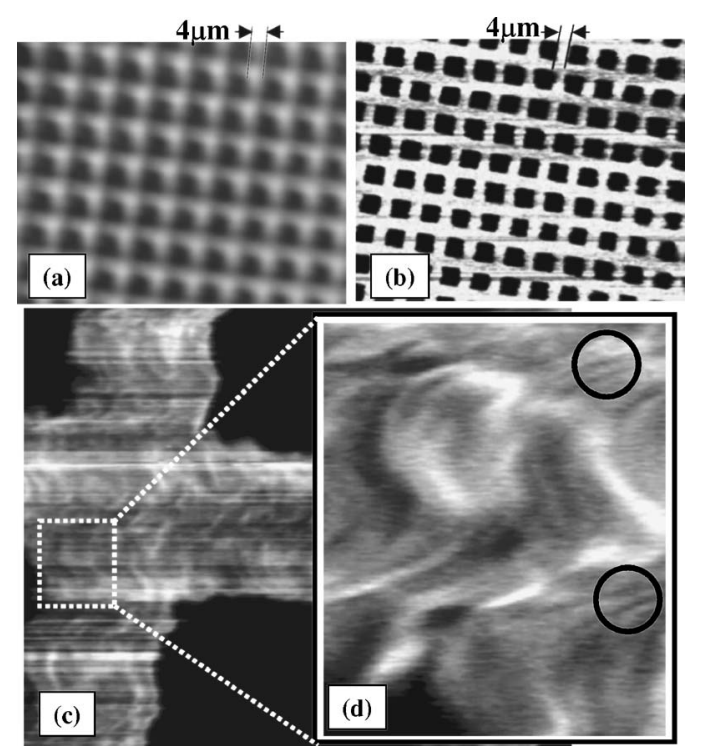

FIG. 4. SEM images of a copper mesh $(4 \mu \mathrm{m})$ obtained using a (a) TE cathode and a (b) graphite nanoneedle cathode. (c) High-resolution SEM image of the copper mesh obtained using a graphite nanoneedle cathode. 
was smeared because of its large source size. The brightness $B$ was calculated as

$$
B=\frac{I}{d S d \Omega}=\frac{I}{\pi r^{2} \pi \alpha^{2}},
$$

where $I$ is the probe current, $d S$ is the source size, $d \Omega$ is the solid angle, $r$ is the radius of the beam, and $\alpha$ is the open angle of the electron optics. For the TE cathode, the beam diameter $2 r$ was estimated at about $4 \mu \mathrm{m}$ and the measured $I$ was about $0.6 \mu \mathrm{A}$. Therefore, the brightness $B$ of the TE cathode was estimated to be about $2 \times 10^{8} \mathrm{~A} \mathrm{sr}^{-1} \mathrm{~m}^{-2}$, which coincides with the brightness reported for a tungsten filament TE cathode. ${ }^{6}$ This $B$ value of the TE cathode shows that this method is appropriate for estimating $B$. Figure 4(b) shows the SEM image of the copper mesh obtained using the etched graphite rod. The spatial resolution was clearly improved compared to that obtained using the tungsten filament TE cathode. This result shows that a SEM image was obtained using the etched graphite rod despite a high residual pressure on the order of $10^{-6}$ Torr. However, as shown in Fig. 4(b), many horizontal noise lines were observed. This is because the electron beam fluctuation occurred during the scanning of the electron beam to obtain SEM images, which is known to be a step and spike noise due to ion bombardment and/or atom adsorption. ${ }^{7-9}$

Figure 4(c) shows a higher-resolution image of the copper mesh. Copper grains are clearly evident. For this SEM system, the magnified view (right image) was the highestresolution image obtained using the graphite nanoneedle cathode. The width of each pixel in this digital image (right image) corresponds to $9 \mathrm{~nm}$. Based on this result, the maximum spatial resolution of this SEM optical system equipped with a graphite nanoneedle cathode was estimated to be $30 \mathrm{~nm}$ (indicated by circles); therefore, the virtual source size $(d S)$ was estimated to be $160 \mathrm{~nm}$. This value of virtual source size coincides with the diameter of graphite nanoneedle; thus, the image was obtained by a single graphite nanoneedle. The electron beam current measured by the Faraday cup was about $30 \mathrm{nA}$. Based on these experimentally determined parameters (i.e., spatial resolution, $d S$, and $I$ ), $B$ of the graphite nanoneedle cathode was on the order of $10^{11} \mathrm{~A} \mathrm{sr}^{-1} \mathrm{~m}^{-2}$, which is almost the same as that of CNTs. ${ }^{3}$ Because the SEM system using a graphite nanoneedle had no focusing electrode, such as a suppressor, the electron beam was spread over a wide angle. A much higher brightness should be attainable, when we make a crossover in front of the cathode in order to reduce a source size and increase a current in the same solid angle.
Compared to other FE cathodes, such as a tungsten tip, a field emitter made of carbon material has many advantages. The first advantage is that the sticking probability of $\mathrm{CO}$ and $\mathrm{H}_{2}$ on a carbon surface is much less than that on a metal surface. $^{7-9}$ The second advantage is that the electric field for electron emission can be decreased, and thus the effect of ion bombardment is reduced because the work function for carbon is smaller than that for tungsten metal. The third advantage is that the etched graphite surface is similar to a bundle of nanoneedles, and thus makes the cathode more durable compared to a single emission source CNT cathode. This is because, although the high residual pressure causes both ion bombardment and momentary discharge of the FE cathode, leading to damage of the CNT cathode, a bundle of graphite nanoneedles provides many emission sites that thus add durability against residual gas atoms.

The SEM images were acquired within $5 \mathrm{~s}$ to avoid stepspike noise ${ }^{7-11}$ in the images. To obtain clearer images, both the step and spike noises must be reduced. Several methods to reduce step-spike noises, including (a) reduced residual pressure, (b) lower probe current operation, (c) lower voltage operation, (d) use of a series resistance to the cathode, and (e) heating of the graphite nanoneedle, can be applied to achieve high-resolution clear SEM images at high residual pressure.

In conclusion, a graphite nanoneedle fabricated by a simple etching technique yields an intense electron emission source at high residual pressure with brightness on the order of $10^{11} \mathrm{~A} \mathrm{sr}^{-1} \mathrm{~m}^{-2}$. The performance of this cathode was demonstrated by the fabrication of SEM at a high residual pressure of $10^{-5}-10^{-6}$ Torr. This graphite nanoneedle can be applied to sophisticated devices, such as microfocus x-ray generators, as well as to an FE electron microscope system.

${ }^{1}$ J. M. Bonard, J. P. Salvetat, T. Stockli, L. Forro, and A. Chatelain, Appl. Phys. A: Mater. Sci. Process. 69, 245 (1999).

${ }^{2}$ S. T. Purcell, P. Vincent, C. Journet, and V. Y. Binh, Phys. Rev. Lett. 88, 105502 (2002)

${ }^{3} \mathrm{~N}$. de Jonge, Y. Lamy, K. Schoots, and T. Oosterkamp, Nature (London) 420, 393 (2002).

${ }^{4}$ T. Matsumoto and H. Mimura, J. Vac. Sci. Technol. B 23, 831 (2005).

${ }^{5}$ R. H. Fowler and L. W. Nordheim, Proc. R. Soc. London, Ser. A 119, 173 (1928).

${ }^{6}$ D. C. Joy, Scan Electron Microsc. 1977I, 1 (1977).

${ }^{7}$ G. A. Beitel, J. Vac. Sci. Technol. 9, 370 (1972).

${ }^{8}$ S. Yamamoto, S. Hosoki, F. Fukuhara, and M. Futamoto, Surf. Sci. 86, 734 (1979).

${ }^{9}$ S. Hosoki, S. Yamamoto, M. Fukumoto, and S. Fukuhara, Surf. Sci. 86, 723 (1979).

${ }^{10}$ F. S. Baker, A. R. Osborn, and J. Williams, J. Phys. D 7, 2105 (1974).

${ }^{11}$ C. Lea, J. Phys. D 6, 1105 (1973). 